\title{
Política internacional en América Latina: seguridad en los primeros años del decenio de 1980 y después*
}

\section{INTRODUGCIÓN}

Los acontecimientos que dan forma a la política internacional o influyen en ella son tanto antiguos como nuevos. Clasificarlos puede ser difícil y establecer causalidad puede serlo incluso más. De este modo, para los fines del presente artículo adaptaré el refrán inglés y consideraré no sólo que los viejos pecados tienen largas sombras sino que también las tienen los nuevos. Además, la política internacional es fastidiada por una teología curiosa en el sentido de que no son solamente los pecados de un individuo los que pueden enviarlo al cielo o arrojarlo al infierno, sino que a la larga, los pecados de otro actor pueden ser más importantes en la definición del destino de un actor determinado.

En el caso de América Latina ${ }^{1}$, sería imposible considerar o únicamente aquellos acontecimientos actuales y de los últimos años como los que están configurando la forma de la política internacional en la región en lo relativo a los aspectos de seguridad, o aquellos respecto de los cuales son responsables las naciones -es decir, los actores políticos colectivos- de la región.

En primer lugar, en la política internacional la cronología no es el factor más importante, es decir, los acontecimientos que tuvieron Iugar hace mucho tiempo pueden tener mayor influencia que los comparativamente recientes. En segundo lugar, los acontecimientos demoran en cristalizar desde el punto de vista del tipo y grado de influencia que tendrán en los acontecimientos políticos

\footnotetext{
*Documento preparado para presentarlo a la vi Reunión Anual del RIAL, celebrada en El Colegio de México, México D. F., 26 al 28 de noviembre de 1984.

${ }^{2}$ Hago uso de la expresión América Latina con algunas reservas. Hay una discusión en cuanto a si la expresión es aplicable y si existe tal cosa llamada América Latina. No deseo entrar en esa discusión, ya que doy por sentado que comprende temas que no corresponden a este artículo; sin embargo, para los fines de las cuestiones relacionadas con la seguridad y la paz, es imperiosamente necesario dividir a "América Latina" en, por lo menos dos entidades: América del Sur y América Central.
} 
e internacionales en un campo tan variado como el que se está examinando. De este modo, a mi juicio, tiene más sentido examinar un conjunto de acontecimientos que han tenido lugar en años recientes, y que continúan influyendo de manera significativa en la política internacional de la región, y seguir agregando otros acontecimientos en la medida en que sigan influyendo en la política internacional de la región, o en la medida en que cambie su grado de influencia.

Con motivo de la $v$ Reunión Anual del RIAL celebrada en Buenos Aires en noviembre de 1983, presenté un documento titulado "Security in South America post-MIalvinas (or post-Grenada?): Strategic and Military Implications"2. El presente artículo constituirá un intento de actualizar algunas de las ideas desarrolladas en ese trabajo anterior.

Desde el punto de vista de la paz y la seguridad, es probable que los acontecimientos úmicos o múltiples, más recientes o más antiguos, que pueden contribuir de manera más significativa a la conformación de la política internacional en América Latina sean los siguientes, en el orden en que se presentan:

I) El comportamiento internacional de los Estados Unidos, especialmente con respecto a la Unión Soviética;

2) EI comportamiento de la Unión Soviética con respecto a los Estados Unidos;

3) La medida en que estos dos actores estén o no estén dispuestos, en el.curso de su acción recíproca, a continuar "transfiriendo" los motivos de sus controversias al territorio de las Américas;

4) Las consecuencias de la guerra de las Malvinas sobre las élites militares y civiles de las Américas, especialmente en América del Sur;

5) La invasión y conquista de Granada (u éste es un conjunto de la acción recíproca entre las Grandes Potencias);

6) La cuantiosa deuda externa de los países más importantes de América Latina con los bancos privados de los Estados Unidos, y las repercusiones que esa situación puede tener en las élites civiles y militares de los países del continente, para no mencionar las consecuencias que ya está teniendo sobre las masas;

7) La durabilidad o no durabilidad de Ios procesos de redemocratización por los que están atravesando varios países del continente;

-Véase Alexandre de S. C. Barros, "Security in South America post-Malvinas (or post-Grenada?): Strategic and Military Implications", documento presentado en la Reunión Anual del RIAx, celebrada en la Universidad de Belgrano, Buenos Aires, Argentina, del 22 al 25 de noviembre de 1983. 
8) La necesidad de examinar y, a la larga, redefinir el papel de los militares en los países de América del Sur y América Central (y éste es un subconjunto del proceso de redemocratización);

9) La creciente importancia del desarrollo de la producción de armas en países del continente, sin que sean necesariamente controlados por los Estados Unidos, como solía ocurrir cuando los Estados Unidos mantenían el casi monopolio del suministro de armas a los países de las Américas ${ }^{3}$.

${ }^{3}$ Sobre la cuestión deI control por parte de los Estados Unidos respecto de las ventas de armas a sus aliados de las Américas, véase Alexandre de S. C. Barros, "The Diplomacy of National Security: South American International Relations in a Defrosting World", en Latin America: The Search for a New International Role, editado por Ronald G. Hellman y H. Jon Rosenbaun (Beverly Hills, California, Sage: 1975), pp. 131-151, y también Jack Child, "The InterAmerican Military System", Tesis de Doctorado, American University, 1978. Una versión más resumida de la tesis de Child es Unequal Alliance: The Inter-American Military System, 1938-1978 (Boulder, Colorado: Westriew Press, 1980). La cuestión de la producción de armas implica varios detalles técnicos que tienen consecuencias politicas muy inmediatas para los paises sudamericanos, especialmente para la Argentina y el Brasil, los dos países que poseen las industrias de armamentos más desarrolladas del continente. En algunos casos, la tecnología de la producción de armamentos -especialmente cuando dicha tecnologia proviene de los Estados Unidos- es un producto muy controlado, especialmente en lo que se refiere a las reexportaciones. La consecuencia de esto es que las exportaciones de armas desde un país como el Brasil o la Argentina pueden ser por último controladas por el proveedor de tecnologia -o de algunas partes del arma final o del sistema de armamentos- si el proveedor así lo desea. Desde el punto de vista militar esto vincula necesariamente la política exterior del país donde se efectúa el montaje al "proveedor de tecnologia" o al "proveedor de partes", con lo que se limitan severamente las opciones del "pais de montaje". Si consideramos que en el caso de América del Sur uno de los proveedores potenciales de tecnologfa respecto de varias cosas, incluidos los armamentos, son los Estados Unidos, es significativa la posibilidad de que las restricciones impuestas por los Estados Unidos lleguen a determinar la polftica internacional de los paúses menos desarrollados. Si consideramos, por ejemplo, el caso del Brasil, que ha venido realizando en los últimos años grandes esfuerzos para desarrollar una industria de armamentos, que dicho sea de paso está logrando un éxito relativo en cuanto a las exportaciones, el mero hecho de que los Estados Unidos tenga tantos intereses en tantas partes del mundo puede limitar considerablemente las opciones del Brasil con respecto a sus exportaciones de armamentos. El aspecto ético de las exportaciones de armamentos es actualmente más bien "desconcertante" en el Brasil, y respecto de él los liberales todavía no han podido ponerse de acuerdo. Ello ocurre porque se lo está considerando como tuente de orgullo nacional. Además, en una situación de crisis de deuđa, el solo hecho de que haya un sector de la economia que sea capaz de producir ingresos en divisas de una manera más bien exitosa (y los ingresos estimados de las exportaciones brasileñas son de alrededor de 2-3 mil millones de dólares para 1984) es causa de alguna confusión para muchas personas que pueden tener dudas éticas acerca de las exportaciones de armamentos. Otra consecuencia importante del desarrollo de esa industria es que los sindicatos de la región donde están situadas la mayoría de las fábricas de armamentos -São José de Campos, cerca de São Paulo- están desarrollando también una mentalidad política muy 
10) $Y$, finalmente, la existencia en la región de rivalidades históricas $\mathrm{y} \cdot \mathrm{de}$ conflictos fronterizos no resueltos $\mathrm{o}$ pendientes pero que se mantienen latentes.

\section{ALGUNOS ANTECEDENTES: LA SEGURIDAD Y LAS GRANDES POTENCIAS}

El decenio de 1970 se caracterizó por un proceso de descongelamiento de las relaciones entre las Grandes Potencias y algunos de los aspirantes a esa condición.

El Gobierno de Nixon, el breve interregno representado por la presidencia de Ford y el Gobierno de Carter fueron periodos durante los cuales los Estados Unidos no enfrentaron abiertamente a sus grandes rivales en el plano internacional. Además de las relaciones entre las potencias más importantes, el derrocamiento del gobierno de Salvador Allende en Chile, las dos crisis del petróleo y la "caída" del Irán fueron Ios acontecimientos más importantes de la política internacional4.

Con respecto a América del Sur y América Central, las dos crisis del petróleo no produjeron ningún efecto en el continente que fuese peculiar o diferente de lo que ocurrió en otras regiones del mundo. La diferencia radicó en el grado de la repercusión y no en su naturaleza.

El caso del derrocamiento del gobierno de Ia Unidad. Popular en Chile fue diferente en el sentido de que el apoyo prestado por los Estados Unidos a esa acción creó una cicatriz profunda y visible en la política del continente. La medida de los Estados Unidos fue adoptada, por una parte, teniendo presente la posibilidad del aumento de la influencia rusa en el continente $y$, por otra parte, considerando la verdadera amenaza representada por la existencia de un gobierno socialista en las Américas.

Aparte de ése, pocos acontecimientos importantes de política internacional durante los años setenta afectaron directamente a la política internacional en América Latina, así como a la relaçión del continente con su socio más importante, los Estados Unidos.

Hablando en términos económicos, durante estos años el mundo perdió un polo dominante ${ }^{5}$. Hablando en términos generales, la

insólita para las normas brasileñas. Ello es así en el sentido de que los trabajadores de esta región están llegando a ser más orientados hacia el "belicismo" que lo que cabría haber esperado de los sindicatos del Brasil.

'La revolución del Irán es importante desde el punto de vista de la polftica internacional debido, entre muchos otros factores, a que frustró tanto las inversiones políticas de los Estados Unidos como las esperanzas de la Unión Soviética, es decir, ninguna de las dos Grandes Potencias consiguió su propósito de sacar provecho de la cafda del Sha.

'Una evaluación muy concisa y objetiva de la situación económica $-y$, hasta cierto punto, general- del mundo con respecto a la falta de un polo dominan- 
"división de poderes" que los Estados Unidos deseaban hacer con sus principales aliados dio origen a la idea de una multipolaridad, para ser preciso con el establecimiento de cinco centros de poder, a saber, los Estados Unidos, la Unión de Repúblicas Socialistas Soviéticas, China (que tenía una situación internacional más elevada debido a su reconocimiento por parte de los Estados Unidos), Europa Occidental y el Japón.

A pesar de estos cambios en el mundo en su totalidad, América del Sur y América Central siguieron relativamente inalteradas por esos cambios, es decir, la división del mundo de los días del punto culminante de la Guerra Fría siguió prevaleciendo en el continente. Algunos países trataron de aligerar el control ejercido por la potencia hegemónica de la región -los Estados Unidos- mediante la práctica de una "diplomacia de seguridad nacional", pero esos esfuerzos no condujeron realmente muy lejos ${ }^{6}$.

Gon respecto a este tema, la toma de posesión del Gobierno de Carter no introdujo necesariamente ninguna nueva tendencia, sino que simplemente aceleró algunas que habían estado presente durante algún tiempo. Esto fue así, a pesar de la apariencia de cambio producida por Carter?

La toma de posesión del Gobierno de Reagan fue el factor político más importante que afectó a América Latina en el plano internacional. A diferencia de algunos de sus predecesores, Reagan no se limitó a "reaccionar" ante los acontecimientos, como lo había hecho Jimmy Carter, ni trató de introducir más "igualdad entre semejantes" en el mundo, como había ocurrido en el Gobierno de $\mathrm{Ni}$ xon, durante el cual el marco conceptual que guió la política exte-

te y la falta de hegemonía fue hecha por el economista brasileño María de Conceiçao Tavares durante la "Jornada de Debates Estados Unidos-América Latina", realizada en el Centro de Estudios Sumaré, con el patrocinio del Instituto de Relaçoes Internacionais de Pontificia Universidade Católica do Rio de Janeiro, 23 de agosto de 1984 . En varias partes del presente artículo utilizaré libremente opiniones sustentadas por María de Conceiçao.

"Sobre la "diplomacia de seguridad nacional" practicada por los países de las Américas, véase Alexandre de S. G. Barros, "The Diplomacy of National Security: South American International Relations in a Defrosting World", en Latin America: The Search for a New International Role, editado por Ronald G. Hellman y H. Jon Rosenbaum (Beverly Hills, Galifornia, Sage: 1975), pp. 131 a 151 .

'Es importante tener presente que el Gobierno de Garter fue sumamente intervencionista con respecto a los pafses latinoamericanos. Esta perspectiva rara vez la pierden aquellos grupos que fueron beneficiados por las intervenciones del Gobierno de Carter. Independientemente de las preferncias o antipatías polfticas, ideológicas y éticas, la naturaleza de la intervención del Gobierno de Carter no fue diferente de la realizada por otros gobiernos, republicanos o demócratas. 
nor de los Estados Unidos fue el del Secretario de Estado Henry Kissinger.

Muy por el contrario, Reagan adoptó iniciativas -aun cuando algunas de ellas únicamente a los niveles retóricos y de asignaciones presupuestarias para la producción de armamentos- que produjeron efectos importantes tanto en el plano económico como en el militar.

Desde el punto de vista económico, Reagan puso en claro con su política económica que los Estados Unidos recuperarían su hegemonía económica. Incluso a costa de una importante recesión interna (y huelga subrayar que a costa de una importarite recesión internacional). La política económica adoptada durante su gobierno reestableció la hegemonía del dólar como la moneda más importante del mundo. Amigos y enemigos por igual se vieron obligados a seguir a los Estados Unidos, tuvieran o no tuvieran deseos de hacerlo.

Militarmente hablando, el equilibrio entre los Estados Unidos y la Unión Soviética no experimentó cambios significativos, es decir, aunque los Estados Unidos no realizaron progresos sustanciales en cuanto a esferas de influencia, no cedió ante la Unión Soviética ni ante "convidados de piedra" en la política internacional tales como fue el caso con motivo de la revolución irani".

De este modo, resumiendo, la política adoptada por el Gobierno de Reagan en el campo internacional, y especialmente en lo que es pertinente para América Latina, se centró en los siguientes aspectos:

1. Recuperación de la lealtad y de la alineación económica de importantes aliados, tales como el Japón, Alemania Occidental (y otros países europeos), Canadá y México;

2. Reafirmación de la hegemonía militar de los Estados Unidos en el mundo para impedir cualquier avance por parte de la Unión Soviética en cualquier lugar del mundo;

3. Cuantiosa inversión en complejos sistemas de armamentos, para impedir que la Unión Soviética sea capaz de continuar compitiendo, debido a su presunta incapacidad económica y tecnológica para competir;

4. Fomento de la integración con América Central, por cualquier

${ }^{\text {s}}$ Sobre la cuestión de la realinación de los aliados de los Estados Unidos con las políticas económicas de Reagan, véase "The Europeans Come Over to the Supply Side", en Business Week (International Edition), No 2862-192, 18 de octubre de 1984, pp. 26 y 27.

${ }^{2}$ Estoy en deuda con Jefferson Barros por discusiones acerca de esta cuestión. 
medio que se estime necesario (y la invasión de Granada constituyó sólo un ejemplo de hasta qué punto estaba dispuesto a llegar el Gobierno de Reagan para lograr sus objetivos) ${ }^{10}$.

Además, existen varios puntos importantes que tienen que ser tenidos en cuenta. En la fecha en que se está escribiendo el presente artículo -octubre de 1984- entre las varias posibilidades futuras, la más probable es la reelección del presidente Reagan; de este modo, además de las medidas muy "objetivas" relacionadas con América del Sur y América Central en los campos militar y político, hay algunas otras que al parecer es probable que se produzcan, las cuales ciertamente tendrán consecuencias en los aspectos de seguridad.

1. El Gobierno de Reagan está comprometido con la aplicación de un modelo económico basado en el libre comercio, el libre flujo de capital y en última instancia la libre empresa en el mundo en su totalidad; de no ser ello posible, este modelo se aplicará, por lo menos, en la parte del mundo que está a su alcance. La exportación de capital y de conocimientos especializados de los decenios de 1950, 1960 y 1970 ahora permite a los Estados Unidos comprar prácticamente todo lo que necesita para el funcionamiento de su economía y toma en consideración la transición que es probable que tenga lugar en los próximos años hacia la "terciarización" de la economía de los Estados Unidos. Por una parte, esto hace a los Estados Unidos más dependiente del mundo, pero también hace que sea más probable que adopte políticas más duras encaminadas a preservar sus intereses en todo el mundo.

Por lo que se refiere a América Latina, la menor dependencia de las relaciones multilaterales y la mayor dependencia de las relaciones bilaterales es el más evidente. Huelga decir que esto ofrece ventajas para los Estados Unidos en el caso de fricción "de bajo nivel", pero también aumenta la posibilidad de fricción de "alto nivel". Hasta ahora, los Estados Unidos han limitado sus políticas firmes sobre relaciones multilaterales a organizaciones internacionales no relacionadas estrictamente con asuntos de seguridadi1. Sin embar-

${ }^{10}$ Se puede discutir que la elección de Granada como "caso ejemplarizador" para fijar el estilo de la relación que el Gobierno de Reagan deseaba introducir en sus transacciones con la Unión Soviética en el hemisferio occidental ha sido una elección "cobarde", una "empresa fácil" (o cualquiera otra expresión que se quiera escoger para dar la idea de que la diferencia entre los Estados Unidos y Granada era absurdamente grarrde); sin embargo, el hecho simple que no se puede pasar por alto es que las naciones, igual que los individuos, tratan de acrecentar al máximo sus ganancias y reducir al mínimo sus costos, y en ese sentido Granada constituyó "una elección perfecta" para expresar el mensaje que el Gobierno de Reagan deseaba transmitir.

IILa política del Gobierno de Reagan en cuanto a restarle importancia a la ayuda $y$ las relaciones multilaterales ya se ha sentido en sus políticas respecto de la Oficina Internacional del Trabajo y la UNEsco. El tercer conflicto que se 
go, dado que es muy probable que el Presidente Reagan sea reelegido, tal vez sea oportuno preguntarse: ¿qué vendrá luego? No es imposible considerar que puedan producirse semejantes retiros, los que reducirían espectacularmente la eficacia de las organizaciones multilaterales que han contribuido en el pasado reciente a evitar conflictos $^{12}$.

\section{SEGURIDAD Y CONFRONTACIÓN LOGAL}

El culpar única y exclusivamente a las Grandes Potencias por los males que puedan atormentar a América del Sur y América 'Central en cuestiones de paz y seguridad puede ser cómodo, pero constituye ciertamente el resultado de la mala memoria o de la falta de perspicacia.

está planteando én esta materia es con el Banco Mundial y más concretamente con la Asociación Internacional de Fomento, Sobre la controversia entre los Estados Unidos de América y la Asociación Internacional de Fomento, véase "Reagan vs. the World Bank: Showdown Time", en Business Week (International Edition), No 2862-192, 19 de octubre de 1984, pp. 16 y 17.

${ }^{10 A I}$ respecto, es importante señalar que el posible retiro de los Estados Unidos de otras organizaciones internacionales- especialmente las que se ocupan de la seguridad- no aumenta necesariamente el riesgo de hecho de una confrontación directa entre las Grandes Potencias por la sencilla razón de que hay tanto en juego en su relación. Sin embargo, puede aumentar considerablemente el riesgo de una confrontación indirecta entre las Grandes Potencias mediante guerras de interés para ellas ya sea en territorio de países del Tercer Mundo o a expensas de esos países. Un pensador militar que considera que esta es la opción más probable para el despliegue de las fuerzas militares de los Estados Unidos es el ex Director de la CIA, Almirante Stansfield Turner: "Al mantener sus efectivo militares, hoy en día los Estados Unidos tienen en cuenta tres amplios objetivos: 1) disuadir de la guerra nuclear o estar listos para librar esa guerra si falla la disuasión; 2) ayudar a la defensa de Europa Óccidental y de Corea del Sur, las zonas donde tenemos estacionadas fuerzas de combate estadounidenses; y 3) poder intervenir en otras regiones del mundo si nuestros intereses exigen dicha intervención. (...). Finalmente, llegamos a la intervención en el Tercer Mundo. Desde la Segunda Guerra Mundial, seis veces hemos combatido en esas regiones. Supongo que es probable que lo hagamos de nuevo, ya que la inestabilidad de los países en desarrollo probablemente será por lo menos tan generalizada en los próximos años. Nuestro defíciente desempeño en tres de esas situaciones de combate indica nuestra falta de preparación para ese tipo de guerra. Estas suposiciones me llevan a concluir que nuestra necesidad más urgente es la de estar mejor preparados en el campo en que es más probable que seamos desafiados, a saber, en la intervención alrededor del mundo. La disuasión de la guerra nuclear, aunque constituye nuestro objetivo más vital, aparece en segundo lugar en mi lista porque, en este campo, ya estamos bien preparados. De este modo, fijaria mis prioridades en la siguiente forma: intervención, primero; disuasión nụclear, segundo; y defensa de Europa Occidental y Corea del Sur, tercero". Stansfield Turner, "A Strategy for the 90's", New York Times Magazine, mayo de 1984 (resumen de un artículo que se publicará en The Art and Practice of Military Strategy, editado por el Capitán George Thibault, Washington, D. C;; National Defense University, 1984). 
Durante èl punto máximo de la guerra fría esa pudo ser una postura realista. Alrededor de mediados del decenio de 1970 los conflictos relativamente pertinentes en América Latina sin la participación o inspiración de las Grandes Potencias liegó a ser una posibilidad teórica ${ }^{13}$. Dicha teoría se materializó sólo en 1982, cuando Argentina intentó recuperar las Malvinas. La importancia simbólica de dicha iniciativa argentina radica en el hecho de que hizo posible poner fin al "paradigma"11 que prevalecía en América Latina, a saber, que la "guerra autónoma" no era una posibilidad viable en el continente. Una vez que ella ocurrió, el riesgo se hizo permanente. De ahora en adelante no hay ninguna "tradición", "creencia" o cualquier pensamiento o doctrina similar que pueda actuar como disuasivo o limitación para que los países latinoamericanos vayan a la guerra. De ahora en adelante se podrá considerar como un asunto de decisiones bien pensadas $y$ bien informadas -es decir, meterse en un conflicto bien calculado- o decisiones deficientemente informadas, tal como ocurrió con la tentativa argentina en el caso de las Malvinas, pero ya no hay más una "barrera sicológica"15.

Una vez que estas limitaciones doctrinarias hayan perdido su fuerza, en ese caso lo que es importante en el contexto de posibles conflictos en el continente es buscar donde es probable que ellos ocurran debido a "animosidades" existentes o en formación, y los tipos de condiciones materiales y políticas que permiten que los

\footnotetext{
${ }^{1}$ Sobre este tema, véase Alexandre de S. C. Barros, "Regional Rivalries and War Probabilities in South America", documento presentado en la Reunión Anual del Seminario Inter-Universitario sobre Fuerzas Armadas y Sociedad, celebrada en la Universidad de Chicago, 23 a 25 de octubre de 1980.

${ }^{{ }^{4}}$ Sobre paradigmas, véase Thomas S. Kuhn, The Structure of Scientific Revolutions, Ghicago: The University of Chicago Press, 1970 (segunda edición).

IJ Una de las instituciones importantes en este esquema fue la Escuela de las Américas, que funcionaba en Panamá, con la inspiración y el patrocinio de los Estados Unidos. La Escuela estaba encargada de capacitar y socializar a oficiles militares de América Latina en técnicas militares y valores relacionados con su papel en el contexto de la fuerza fría. En América Latina esto podía traducirse por "seguridad interna", "actividades antiguerrilleras", etc. La consecuencia de este tipo de capacitación y socialización fue que las doctrinas y valores impartidos por la Escuela actuaron, de una u otra manera, como un factor para disminuir la posible orientación de los oficiales militares latinoamericanos hacia la guerra externa dentro del continente, ya que eso no le interesaba a los Estados Unidos. La Escuela fue cerrada en septiembre de 1984, como uno de los resultados de la reorganización de la relación existente entre los Estados Unidos y Panamá. Hay rumores de que se establecerá otra Escuela equivalente, tal vez en Honduras. Todavfa es incierto si se hará dicha tentativa. Entretanto, si consideramos que la medicina de un hombre es veneno para otro, la falta de la capacitación y socialización proporcionadas por la Escuela puede perfectamente disminuir el número de militares latinoamericanos obsesionados con la seguridad interna - Io que tendría repercusiones en los futuros gobiernos militares del continente- $y$, al mismo tiempo, permitir que crezca el apetito de los militares latinoamericanos por la guerra externa en el-continente.
} 
conflictos tengan Iugar, a saber, la existencia de armamentos y fuerzas para hacer la guerra.

En América Latina, la existencia de fuerzas y de armas para hacer la guerra es un factor importante que está cambiando. Como señalé anteriormente, mientras los Estados Unidos mantenían el cuasimonopolio sobre el suministro de armas a la región más la hegemonia política, les era posible mantener el control sobre la corriente de medios físicos para hacer la guerra. Además, la existencia de hegemonía política y el importante papel desempeñado por los Estados Unidos en el suministro de doctrina y formación militar contribuian a impedir la existencia de las condiciones "sicológicas" para hacer la guerra ${ }^{16}$.

Sin embargo, las cuestiones de la existencia de las orientaciones de doctrina y los medios físicos para hacer la guerra no es la única cuestión, ni, tal vez, la principal. Así ocurre porque la capacidad que tienen los gobiernos para enviar a la población de sus países a la guerra parece ser muy elevada, a pesar de posibles situaciones de "bajo grado de ciudadanía", como el que habitualmente caracteriza a la mayoría de los países latinoamericanos. Si se agrega a esto el importante papel que puede desempeñar el patriotismo, incluso en una situación que mirada desde el exterior parezca absurda -tal como la de unión nacional para respaldar la guerra durante los días del Gobierno de Galtieri en la Argentina- en ese caso es perfectamente posible imaginar que esas situaciones se repitan en el continente en el futuro próximo. El hecho de que hubiera una comisión investigadora como la Comisión Rattenbach no constituye necesariamente un seguro contra la repetición de tales acontecimientos debido a una tendencia intrinseca de las organizaciones burocráticas dé ser incompetentes para aprender de sus actos, tendencia que se hace incluso más pronunciada en el caso de las organizaciones autoritarias ${ }^{17}$.

No cabe duda alguna de que las animosidades no se terminan o

${ }^{19}$ Sobre esta cuestión, véase Alexandre de S. G. Barros, "The Diplomacy of National Security: South American International Relations in a Defrosting WorId", en Latin America: The Search for a New InTternational Role, editado por Ronald Hallman and F. Jon Rosenbaum (Beverly Hills, California: Sage 1975).

${ }^{1 T}$ Para un examen de la incapacidad de las organizaciones burocráticas para aprender como resultado de sus propios errores, véase Michel Crozier, The Bureacratic Phenomenon (Chicago: University of Chicago Press, 1964). Sobre la inclinación de las organizaciones autoritaxias a exacerbar esta tendencia, Norman F. Dixon señala que: "Es una triste característica de las organizaciones autoritarias que su naturaleza inevitablemente milite contra la posibilidad de aprender de la experiencia mediante el reparto de la culpa. La razón no es tan difícil de encontrar. Dado que el autoritarismo es él mismo el producto de de. fensas sicológicas, las organizaciones autoritarias son expertas en desviar la culpa. Lo hacen mediante la negación, la racionalización y la búsqueđa de víctimas propiciatorias o por alguna mezcla de las tres. Como quiera que se logre, el re- 
se borran de la noche a la mañana; la negociación internacional es importante, pero también lo son la socialización de las élites y de las masas. En otras palabras, esto significa que una controversia que es arreglada mediante un tratado no se resuelve automáticamente y para siempre; puede ser revivida si se dan las condiciones políticas apropiadas como una posible fuente de conflicto. En el caso de América Latina, en las palabras de un funcionario del gobierno del Brasil, "había una tradición de solucionar las controversia internacionales mediante tratados y negociaciones"18.

Sin embargo, esta "tendencia" ha sido rota por la Guerra de las Malvinas y existen actualmente varios "puntos críticos" en América del Sur que podrían, en cualquier momento, estallar en un conflicto armado. La interrupción de esta tendencia de evitar la guerra es un hecho que debería hacer que nos preocupáramos por la permanencia en el tiempo de arreglos tales como el del Canal del Beagle.

Esto es así porque el hecho de que dos gobiernos solucionen un problema en una forma que es satisfactoria para ellos en un determinado momento de la historia no constituye suficiente garantía de que el arreglo seguirá siendo satisfactorio para "la nación" o "el universo politico" o "el estado" (como una entidad más permanente). A la larga, esta diferencia entre el interés del gobierno como algo que existe y es válido en cualquier momento determinado, y otros tipos de intereses que, a mediano y largo plazos, pueden superarlos es un punto muy pertinente que hay que tener en cuenta, porque siempre es posible revivir tales animosidades ${ }^{19}$.

sultado neto es que jamás hay una admisión real de fracaso o incompetencia por parte de aquellos que son realmente responsables, por lo que nada se puede hacer para impedir que el hecho se vuelva a repetir". Norman F. Dixon, On the Psychology of Mililary Incompetence (Londres: Futura Publications, 1976), pp. 43 y 44.

${ }^{18} \mathrm{El}$ problema, en este caso, puede consistir en que esta interpretación implique una mezcla de localismo con falta de una visión histórica a más largo plazo. Implica Iocalismo en el sentido de que posiblemente se estaba refiriendo al hecho de que el Brasil podía solucionar sus problemas con sus vecinos mediante tratado; sin embargo, en el Ienguaje directo de un oficial militar brasileño, "decir que nosotros (los brasileños) no tenemos problemas fronterizos es una percepción desde nuestro punto de vista: vayan a preguntarle a nuestros vecinos". Implica falta de visión histórica en el sentido de que pasa por alto el hecho de que la feliz existencia de acuerdos internacionales, negociaciones y órganos multilaterales para impedir el conflicto internacional en América Latina fue el resultado, no necesariamente de un deseo de las élites latinoamericanas sino de las condiciones impuetas a ellas desde el exterior, es decir, la falta de armamentos y las restricciones de su venta por los Estados Unidos.

${ }^{10}$ Esto es especialmente grave en una situación de un grado relativamente bajo de institucionalización, tal como el que caracteriza a varios de los sistemas polfticos en América Latina. Estoy utilizando la idea de institucionalización en el sentido de que un sistema político altamente institucionalizado tiene la capacicad de absorber un cambio - tal como el representado por el arreglo de una controversia fronteriza- y transmitirlo a su élite y a su pueblo, incorporándolo,

$$
\left[\begin{array}{lll}
4 & 2 & ]
\end{array}\right.
$$


- Presentaré a continuación una lista de las animosidades fronterizas históricas que existen actualmente en América Latina, las que pueden ser revividas en cualquier momento, especialmente en una situación en que se rompió la "tradición" de la ausencia de conflicto. Esta enumeración no pretende ser exhaustiva ni el orden particular en que se presentan las controversias pendientes -activaș o latentes- representan intento alguno para ponerlas en un orden jerárquico de las probabilidades de que ocurran. Se trata de una lista que tiene números sólo para hacer más fácil el recuento, sin que haya que atribuirles ningún significado adicional.

El aspecto importante que hay que tener presente con respecto a todas ellas es que, independientemente de su estado activo o latente en términos formales, el hecho concreto es que la mayoría de ellas son cuestiones públicas efectivas o potenciales en la vida política y en la cultura política de los países respectivos. Esta característica de que sean cuestiones públicas hace posible que prácticamente cualquier gobierno pueda hacer uso de este recurso de resolver animosidades, legítimamente o ilegítimamente, a fin de aumentar su apoyo político interno, resarcirse de pérdidas anteriores, o por cualquier otra razón similar. Sin entrar en experiencias extracontinentales o en ejemplos muy alejados en el pasado, sino sobre la base única y exclusivamente de la experiencia del. General Leopoldo Galtieri en el caso de las Malvinas, es perfectamente posible prever que un gabierno que decide hacer uso de este recurso tiene posibidades relativamente buenas de ser apoyado por segmentos considerables de su ciudadanía.

Por último, pero no por ello menos importante, para consternacjón de algunos que sustentan una opinión más optimista, no existe ninguna evidencia o garantías de que medidas irresponsables de esta índole serán adoptadas únicamente o primordialmente por gobiernos militares. Desgraciadamente no existe esa seguridad ${ }^{20}$.

de una u otra manera, en la "cultura política" imperante, o la "autopercepción" de una sociedad, mientras que en una situación de bajo grado de institucionalización este proceso es mucho más difícil de lograr, es decir, el arreglo alcanzado por un determinado gobierno no necesariamente logra ser pasado con facilidad en el transcurso del tiempo, de un gobierno a otro gobierno o de una generación política a otra generación polf́tica. La institucionalización es definida por Samuel Huntington como "el proceso mediante el cual las organizaciones y procedimientos adquieren valor y estabilidad". El nivel de institucionalización de cualquier sistema político puede definirse por la adaptabilidad, complejidad, autonomía y coherencia de sus organizaciones y procedimientos. Para un examen detallado de la idea de institucionalización de Ios sistemas polf́ticos véase Samuel P. Huntington, Political Order in Changing Societies (New Haven, Connecticut: Yale University Press, 1968), pp. 12-24.

${ }^{2}$ Cabe señalar que las recientes confrontaciones fronterizas entre el Ecuador y el Perú tuvieron lugar mientras ambos pafses tenfan gobiernos civiles: Destaco esta situación a fin .de. llamar la atención sobre el hecho de que el supuesto 
Los "puntos críticos" en el continente y su condición actual desde el punto de vista político son los siguientes: ${ }^{21}$.

1) Las Malivinas. A pesar de las conversaciones, evidentemente la cuestión no está solucionada para la Argentina. El hecho de que el actual gobierno esté adoptando medidas para evitar la reaparición del conflicto, si bien es digno de elogios, no resuelve necesariamente el problema para siempre. Es una medida aplaudida del Gobierno de Alfonsín que continúa haciéndolo; sin embargo, las cicatrices dejadas por la prolongada animosidad histórica y por la guerra misma hacen de las Malvinas un punto que ha de ser siempre observado 22 .

2) La controversia del Beagle entre Ia Argentina y Ghile. Esta disputa casi llevó a los dos países a la guerra a fines del decenio de 1970. Ahora hay un arreglo, que es satisfactorio para los dos gobiernos actuales de la Argentina y Chile, lo que es bueno. Sin embargo, el mero hecho de que los dos gobiernos hayan alcanzado un arreglo jurídico no es razón suficiente para creer que la controversia será eliminada definitivamente. En presencia de otras condiciones políticas -internas o externas- es perfectamente posible prever que la controversia puede reavivarse, llegando al punto de un conflicto ${ }^{23}$. Ello puede ocurrir incluso después de la ratificación de

seguro contra la guerra representado por los gobiernos civiles no es sino una ilusión, es decir, las guerras tienen lugar independientemente del carácter -civil o militar- del gobierno.

MEstoy utilizando la expresión "condición política" de una controversia para diferenciarla de "condición juridica". No estoy considerando como factor pertinente si la cuestión está o no está arreglada juridicamente, sino que el punto importante de mi consideración es la importancia que tiene la cuestión en los planos politico $y$ cultural en los países interesados. La tendencia a considerar que una vez que algo está resuelto jurídicamente no es probable que ocurra el conflicto efectivo hace caso omiso de la historia en otras regiones del mundo donde existian las condiciones para el estallido de la guerra, de modo que los tratados $y$ otros instrumentos jurfdicos resultaron ser inoperantes.

^2a denuncia en el Parlamento Británico en septiembre de 1984 de las condiciones en que fue hundido el buque argentino "General Belgrano" constituye un ejemplo de un acontecimiento singular que puede agregar el insulto al daño inferido. Era asunto de público conocimiento hasta septiembre de 1984 que el hundimiento del "General Belgrano" había tenido lugar en "condiciones normales y legítimas de guerra". Con las revelaciones que actualmente están llegando al conocimiento del público, dicha operación tuvo lugar en condiciones tales que se puede poner en tela de juicio su legitimidad, o que se está haciendo. Es muy probable que este acontecimiento y la controversia que está originando permanezcan como una animosidad adicional en las relaciones entre la Argentina y el Reino Unido, lo que contribuirá a impedir que cicatricen plenamente las heridas dejadas por este episodio.

${ }^{3}$ Cabe elogiar el hecho de que el gobierno de Alfonsín, en la Argentina, aparentemente está promoviendo una campaña publicitaria para difundir la noti cia de que se logró el arreglo, y socializar a la población en los nuevos valores,

$$
\text { [ } \left.\begin{array}{lll}
4 & 4 & ]
\end{array}\right]
$$


un tratado por el Congreso, especialmente en una situación en que el Congreso es una institución tan inestable, o después de que un tratado de esta indole ha sido sometido a aprobación mediante plebiscito.

3) Ecuador y Perú. Estos dos países libraron en los años cuarenta una de las pocas guerras que ha habido en América Latina desde la guerra de la Triple Alianza (1865-1870). El conflicto dio por resultado una pérdida considerable de territorio para el Ecuador. El problema ha estado latente, pero en los primeros años del decenio de 1980 hubo pequeños conflictos fronterizos que dejaron en claro que el problema no estaba resuelto para siempre. De este modo, también en este caso, no hay evidencia de que no se producirá un conflicto, sino que todo lo contrario. En este caso particular, las confrontaciones tuvieron lugar mientras ambos pafses tenían gobiernos civiles.

4) Chile, Perú y Bolivia. Este "triángulo" de países protagoniza el litigio más "clásico" del continente. Desde el punto de vista militar y jurídico, la cuestión fue resuelta de hecho con el fin de la Guerra del Pacífico. Sin embargo, la cuestión está todavía muy viva en el sentido de que el arreglo a que se llegó adolecía de un defecto muy grave, cual es la falta de acceso al mar por parte de Bolivia. La cuestión del acceso boliviano al mar es uno de los problemas políticos más presentes en la vida y la cultura políticas de Bolivia. El significado práctico de este tipo de "arreglo" es que prácticamente es imposible considerar que la cuestión no se planteará de nuevo en el futuro en términos conflictivos. Por supuesto, siempre se podrá sostener que Bolivia, por ser un país tan pobre, simplemente no dispondría de los medios económicos y militares para comprometerse en una guerra; sin embargo, no es insólito el caso de países que incluso careciendo de esos medios participan en conflictos, exactamente para llamar la atención sobre sus reclamaciones. De este modo, el problema, tal como fue resuelto por la Guerra del Pacífico, no fue ni es satisfactorio para el Perú ni para Bolivia. El deseo de venganza y de compensación por parte del Perú es también un tema pertinente en la vida política peruana, si bien no tanto como el del acceso boliviano al mar.

5) Los litigios de Venezuela y Colombia respecto del Golfo de Maracaibo. Hasta ahora esta cuestión no ha provocado ningún conflicto en el pasado que pueda invocarse como motivo de venganza o reparación nacionales; sin embargo, el problema existe y no ha sido resuelto todavía.

6) Itaipú como una cuestión entre la Argentina y el Brasil. La

en un intento por dar unavidamás larga al acuerdo (Conversación privada con el corresponsal de un diario. Río de Janeiro, i7 de octubre de 1984). 
construcción de la presa y central hidroeléctrica de Itaipú constituyó, prácticamente durante todo el decenio de 1970, un motivo de desacuerdo entre la Argentina y el Brasil. Actualmente está resuelta, pero estableció algo que es nuevo en América del Sur, a saber, el hecho de que un país tenga intereses directos que están físicamente inmovilizados en el territorio de otro pás ${ }^{24}$. No cabe ninguna duda de que hasta ahora el problema está resuelto, pero estableció una relación original entre países de la región que no estaba presente antes de la construcción de la central de energía hidroeléctrica. Queda por ver en qué forma los países de la región van a actuar y a reaccionar frente a los demás en presencia de esta nueva cuestión; sin embargo, el hecho concreto es que ahora tanto el Brasil como la Argentina tienen intereses creados en la situación del Paraguay ${ }^{25}$. Los tipos de interpretaciones en cuanto a la forma en que se tratarán estas cuestiones pueden cambiar según las personas de que se trate, y pueden variar desde las interpretaciones más sensatas y razonables a las más paranoicas, como la propugnada repetidamente por Paulo Schilling ${ }^{26}$.

HUna situación comparable, sólo por la analogfa, es la que existe entre los Estados Unidos y Panamá, ahora, después del tratado. El Canal está físicamente situado e inmovitizado en territorio panameño y estará bajo el "pleno" control de Panamá; sin embargo, la parte realmente y fundamentalmente interesada en la seguridad del Canal no es Panamá, sino los Estados Unidos.

xaPara un examen detallado de esta cuestión, véase Alexandre de S. C. Barros, "Brazilian Perceptions about Regional Security in Latin America", documento presentado en el Simposio Internacional sobre Seguridad Regional y Paz en América Latina, celebrado en Viña del Mar, Chile, del 19 al 22 de noviembre de 1984.

${ }^{20} \mathrm{H}$ ablando acerca de Itaipú, Paulo Schilling dijo en una conferencia que dictó en la Universidad de Posadas, al norte de Buenos Aires, que Itaipú es una "bomba de agua" que puede inundar a la Argentina. Schilling dijo que "Itaipú fue construida teniendo en cuenta este propósito, aparentemente para hacer frente al desarrollo del programa nuclear argentino. Itaipú está situada a $17 \mathrm{~km}$ de distancia de la frontera argentina. Abrirla podría tener el efecto de 50 bombas nucleares". Según él, la capacidad del lago Ytaipú es de 29 mil millones de metros cúbicos de agua (información dada por $O$ Globo de Río de Janeiro, 6 de octubre de 1984). Para una idea más detallada acerca de las opiniones de Schilling, que han atraido la atención de algunos sectores civiles y militares de la Argentina, véase su libro Brasil va a la Guerra (Buenos Aires: Schapiro Editor (colección Miral), 1974). Las hipótesis básicas de Schilling son, por una parte, que el Brasil actúa como una potencia subimperialista, es decir, Brasil hace lo que los Estados Unidos le dicen que haga; por otra parte, propugna la idea de que el Brasil expandirá su territorio continuando los movimientos de los bandeirantes (los pioneros brasileños que expandieron la frontera hacia el oeste durante los siglos xyII y xvrII). Tal vez las percepciones de Schilling fueron influidas en gran medida por su propia historia personal y política habiendo sido desterrado del Brasil después de 1964 y habiendo vivido en el Uruguay y en la Argentina. Podría argüirse que la opinión de Schilling no concuerda con el paradigma imperante en América del Sur, pero los paradigmas pueden cambiar. Después de todo, ¿̇no creyó la gente durante siglos que la tierra era un disco plano? ¿no creía igualmente que el sol giraba alrededor de 
Esta es sólo una simple lista de las controversias más visibles entre los países de América del Sur que algún día podrían estallar en un conflicto militar. Como dije anteriormente, no es exhaustiva; se trata simplemente de una enumeración para tener en cuenta, pero pueden aparecer otras cuestiones, no necesariamente fundadas en hechos históricos, a saber, cosas relacionadas con cuestiones contemporáneas que pueden generar problemas tales como ocupación de zonas fronterizas, intereses creados en la estabilidad o inestabilidad política de los países vecinos, violaciones de fronteras y apoyo a los grupos que se oponen a los gobiernos de los países vecinos o lucha contra ellos, etc.

\section{UNA NOTA FINAL SOBRE LAS INDUSTRIAS DE ARMAMENTOS SUDAMERIGANAS}

Una cuestión importante que se ha de tener en cuenta -que es probable que sea de importancia relativamente limitada a corto plazo, pero que puede tornarse más importante a medida que transcurra el tiempo- es la del desarrollo de las industrias de armamentos en países sudamericanos, especialmente en el Brasil y la Argentina.

El punto de vista "corriente" acerca de esta cuestión es que el Brasil y la Argentina están creando industrias de armamentos para enfrentarse en una confrontación directa. No hago mía esa opinión, por lo menos no como el tipo más probable de cosa que pueda ocurrir. Mi opinión es que el desarrollo de las industrias de armamentos en esos dos países puede actuar mucho más como un mediọ para influir en el comportamiento de terceras partes en el continente. De este modo, en esta ecuación el Brasil y la. Argentina se tornan mucho más importantes como proveedores de armamentos a otros países que podrían luego utilizar las armas en conflictos unos con otros, que el caso de la confrontación directa entre el Brasil y la Argentina ${ }^{27}$.

Si miramos más adelante en el futuro, otros países pueden también crear industrias de armamentos en el continente, aunque es

la tierra? Para un examen de los paradigmas, véaes Thomas S. Kuhn, The Structure of Scientific Revolutions (Ghicago: The University of Chicago Press, 1970, segunda edición).

arSobre la industria brasileña de armamentos, véase Alexandre de S. C. Barros, "The Brazilian Arms Industry: An Instrument of Foreign Policy", en Arms Production in Developing Countries: An Analysis of Decision-Making, editado por James Katz, Lexington, Massachussets: C. C. Heath \& Co., 1984, Y Alexandre de S. C. Barros, "O Modelo da Indústria Bélica Brasileira: História e Implicaçoes", documento presentado al Simposio sobre los Militares, el Estado y la Sociedad, realizado en la Universidad de Campinas, Sao Paulo, 21 al 23: de marzo de 1984: 
poco probable que en el futuro previsible cualquier país de Sudamérica tenga la capacidad para desarrollar un sistema de produc. ción de armas que pueda tener importantes repercusiones en el continente. Sin embargo, incluso considerando única y exclusivamente el caso de la Argentina y el Brasil, la importancia del fenómeno radica en el hecho de que aparte de las cuestiones "bilaterales" que fueron examinadas en la enumeración que presenté anteriormente en este artículo, desde ahora en adelante dos países de la región están desarrollando sistemas industriales que pueden aumentar en forma sorprendente la complejidad de las relaciones entre los países del continente. Esto es así porque la creciente capacidad industrial de la Argentina y del Brasil en materia de producción de armamentos puede aumentar la posibilidad de que estos dos países traten de jugar con éxito para influir en la región utilizando como fichas su capacidad para suministrar armas ${ }^{28}$.

\section{Una nota final: el Atrántico Meridional}

Un motivo final e importante de preocupación por la seguridad en América del Sur, que es más probable que influya en la Argentina y el Brasil,, es la cuestión de la seguridad del Atlántico Meridional. Es más probable que la importancia del océano afecte directamente a aquellos paises con mayor litoral, a saber, el Brasil.y la Argentina. Sin embargo, si consideramos al Atlántico como una ruta, desde ese punto de vista cualquier problema de seguridad que surja en él ciertamente afectará a todos los países de América del Sur.

En principio, los problemas que es probable que se presenten en el Atlántico Meridional están relacionados con la competencia entre las superpotencias, aunque la evolución de ellos tendería a ser diferente de la que actualmente tiene lugar en América Central. En el presente artículo no entraré en detalles sobre este tema porque sus peculiaridades casi ciertamente exceden los límites de este documento.

En vista de lo anterior, me limitaré a mencionarlo, dando por sentado que es probable que esa cuestión adquiera más importancia en unos pocos años más 29 .

\section{RESUMEN DE LAS GONCLUSIONES}

Por lo que se refiere a la paz y la seguridad, América Latina tiene

${ }^{23}$ Sobre esta cuestión, véase Alexandre de S. C. Barros, "The Brazilian Arms Industry: An Instrument of Foreign Policy", (publicado bajo el tftulo "Brasil") y Jacquelin S. Porth, "Argentina" en Arms Production in Developing Countries: An Analysis of Decision-Making, editado por James Katz, Lexington, Massachussets: D. G. Heath \& Co., 1984, pp. 73 a 88 y 53 a 72 , repectivamente.

${ }^{20}$ Para mayores antecedentes sobre algunas de las cuestiones de seguridad. comprendidas en la cuestión del Atlántico Meridional, véase Alexandre de S. C. 
que dividirse entre América Central y América del Sur, y las cuestiones relacionadas con estos temas son sustancialmente diferentes en las dos regiones. Como señalé en la nota final, el Atlántico Meridional es un tercer tipo de problema, que presenta peculiaridades propias y no es probable que se integre necesariamnte con los otros dos problemas.

Para empezar, es probable que América Central, dada su posición geográfica en relación con los Estados Unidos, sus dimensiones territoriales y su historia pasada, sea una región en la que la mayoría de las cuestiones de seguridad serán sometidas a cuidadoso examen y a la supervisión de los Estados Unidos, es decir, con toda seguridad este último país seguirá una política de intervención -de manera directa o indirecta, abierta o disimulada- en la región.

Esto se debe a que la forma en que cualquier cambio estratégico importante en América 'Gentral puede afectar a los Estados Unidos se percibe como fundamental. En los últimos años, y tal vez en el futuro previsible, el problema clave de América Central es que se convirtió en campo de confrontación entre los Estados Unidos y la Unión Soviética. Si este último país está proporcionando armas a los rebeldes de El Salvador por intermedio de Nicaragua, Cuba u otro país es, desgraciadamente, una cuestión improcedente desde el punto de vista de América Latina. El hecho concreto es que, a pesar de la cantidad y la calidad de la intervención soviética en la región, y no se debe olvidar que hay mucha propaganda de los dos lados participantes en el litigio, el hecho simple es que los Estados Unidos se están ocupando de los problemas en América Central basados en la premisa de que hay intervención soviética allí. ¡Desde el punto de vista del ratón, no tiene importancia si el gato es negro 0 amarillo!

En América del Sur la situación es muy diferente. La distancia desde los Estados Unidos, la historia de la región, el tamaño de los países, etc., crean una situación en que, aunque Sudamérica es geopolíticamente parte de lo que el Departamento de Estado llama Hemisferio Occidental, es mucho menos probable que haya injerencia directa por parte de los Estados Unidos, en comparación con América Central. En esta región hay probabilidades de que los conflictos ocurran de una manera mucho más "autónoma" con respecto a las Grandes Potencias. Como si la escala geográfica y la distancia física no bastaran, tenemos también la disponibilidad de las armas, como señalé anteriormente en el presente artículo.

Barros, "Defense and Security Issues: Implications for the New Atlantic Triangle", documento presentado en la Conferencia " $¿$ Un nuevo triángulo del Atlántico? América Latina, Europa Occidental y los Estados Unidos", celebrada en la Univeridad de Brasilia, 15 al 17 de junio de 1981. 
No obstante, la disponibilidad de armamentos, no es necesariamente una cosa decisiva. Una teoría que existe y que es muy citada cuando se plantea el debate acerca del control de las armas de fuego para los individuos, es que las personas que poseen armas seguramente las usarán si las tienen. Hasta cierto punto esto se puede aplicar a las naciones. Después de todo, las guerras son actos polfticos que se tornan mucho más fáciles de cometer si un país dispone de los medios físicos para respaldar el comportamiento agresivo o belicoso.

En la enumeración de las controversias territoriales existentes en América del Sur que presenté anteriormente, me limité a los problemas definidos bilateralmente, con la excepción de la cuestión trilateral entre Chile, Perú y Bolivia. Sin embargo, debemos tener presente que, cuando los países se desarrollan industrialmente, crecen sus aspiraciones internacionales y desarrollan sus sistemas de transporte y comunicaciones, aumentan casi geométricamente las posibilidades de que las confrontaciones abarquen a más de dos partes. El hecho es que el riesgo está presente, mucho más que en el pasado. Más aún, se está haciendo más y más claro que en determinadas regiones del mundo, cuando las partes interesadas son de cierta escala, la capacidad y el deseo de las Grandes Potencias de intervenir para detener un conflicto disminuye de manera impresionante. Este puede ser un efecto cruel de la "adolescencia", es decir, cuando los países son pequeños y se torna probable que estalle el conflicto, la intervención puede ser fácil y barata para las Grandes Potencias. A la inversa, cuando las proporciones de las partes comprometidas o su ubicación estratégica son de tal magnitud que un conflicto puede alterar o trastornar sustancialmente el statu quo del mundo en su totalidad, en el análisis costo-beneficio de las Grandes Potencias vale la pena pagar el precio político y militar de la intervención.

En el caso de los países "aclolescentes" la situación puede ser diferente y las Grandes Potencias pueden limitar su actitud a la de los bomberos en operaciones en viviendas abandonadas de barrios pobres, a saber, siguen lanzando agua sobre las casas vecinas para que el fuego no se extienda, pero no necesariamente, sofocan el incendio primario. 